\title{
Trasplante combinado hígado-riñón en la fundación valle del Lili, Cali, Colombia - Experiencia de un centro
}

\section{The experience of the Fundacion Valle Del Lili in Cali, Colombia with Combined Liver and Kidney Transplantation}

\author{
Luis Armando Caicedo R., MD, ${ }^{1,3}$ Cristina Vernaza 0., MD, ${ }^{2}$ María Lucía Valderrama M., MD, ${ }^{2}$ Eliana Manzi T., MD, ${ }^{2}$ \\ Gabriel Jaime Echeverri J., MD, 1,3 Diego Fernando Jimenez R., MD, 1,3 Jairo Alberto García A., MD, 1,3 Mauricio Sepúlveda C., MD, 1,3 \\ Liliana Mesa R., MD, 1,3 Juan Guillermo Posada Ch., MD, 1,3 Johana Schweineberg L., MD, 1,3 Oscar Javier Serrano A., MD, 1,3 \\ Jorge Iván Villegas 0., MD, ${ }^{1,3}$ Verónica Botero 0., MD, ${ }^{1,3}$ Carlos Eduardo Durán R., MD,1,3
}

\footnotetext{
1 Unidad de Trasplantes, Fundación Valle del Lili, Cali, Colombia.

2 Centro de Investigaciones Clínicas, Fundación Valle del Lili, Cali, Colombia.

3 CICAT, Centro para la investigación en cirugía avanzada y trasplantes, Facultad de ciencias de la salud, Universidad Icesi, Cali, Colombia.

Fuente de apoyo financiero: no hubo apoyo financiero externo a la Fundación Valle del Lili.

Presentado: XXII Congreso Latinoamericano y del Caribe de Trasplantes. Buenos Aires, Argentina. Diciembre 1-4 2013. Exposición Oral.

Fecha recibido: $21-07-15$

Fecha aceptado: 18-04-16
}

\begin{abstract}
Resumen
Introducción: el trasplante combinado de hígado y riñón (CLK) ha mostrado ser una buena alternativa para pacientes con diagnóstico concomitante de enfermedad renal crónica (ERC) y enfermedad hepática terminal. Algunos estudios han mostrado además un beneficio inmunológico del trasplante combinado, con disminución de la tasa de rechazo del injerto renal.

Objetivo: describir las indicaciones y los resultados clínicos en receptores de trasplante CLK en un hospital de alta complejidad.

Materiales y métodos: se seleccionaron los pacientes con trasplante CLK del registro institucional de trasplante (TRENAL) entre 2000 y 2011. Se describieron las características demográficas y clínicas y se estimó la sobrevida de los pacientes y de los injertos con el método Kaplan Meier.

Resultados: en un período de 11 años, se realizaron 16 trasplantes CLK. Esto corresponde al $1.51 \%$ de los trasplantes de riñón y al $3.48 \%$ de los trasplantes de hígado hechos en la institución durante el mismo periodo de tiempo. La mayoría de los receptores fueron de sexo masculino (10/16). La mediana de la edad fue 56.5 años. La mediana del MELD fue de 17 (RIQ: 12.5-20.5, Rango 8-24). El diagnóstico hepático más frecuente fue cirrosis por NASH (4/16). Todos los pacientes tenían diagnóstico de ERC, en 4 casos secundaria a diabetes mellitus. Las indicaciones más comunes del trasplante fueron ascitis de difícil manejo, encefalopatía recurrente y desnutrición. El tiempo promedio de isquemia en frío del hígado fue de 7,3 horas, y del riñón 9,6 horas. La sobrevida de los pacientes y de los injertos hepático y renal fue de $87,5 \%$ al año y $67,3 \%$ a los 5 años. Cuatro pacientes murieron, 2 casos como consecuencia de sepsis y otros 2 por malignidad.

Conclusión: el trasplante CLK en la Fundación Valle del Lili tiene resultados clínicos satisfactorios y comparables a los reportados en otros centros.
\end{abstract}

Palabras clave

Trasplante combinado hígado-riñón, CLK, sobrevida, rechazo del injerto.

\section{INTRODUCCIÓN}

En 1984 Margreiter reportó el primer trasplante combinado de hígado y riñón (CLK - Combined Liver-Kidney) (1). El trasplante CKL ha mostrado ser una buena alternativa terapéutica para pacientes que cursan con enfermedad hepática terminal y enfermedad renal crónica (2). Basados en los datos de la OPTN (Organ Procurement and Transplantation
Network), entre 1988 y junio del 2014 se han realizado en los Estados Unidos 5816 trasplantes CKL. Las recomendaciones del consenso americano sobre trasplante CLK muestran el beneficio que obtienen los pacientes con CLK, en términos de sobrevida, al compararse con aquellos que reciben solo trasplante hepático con creatinina sérica pretrasplante $>2,0 \mathrm{mg} / \mathrm{dL}$ y están en diálisis (3). Varios estudios han encontrado la capacidad del injerto hepático de 


\begin{abstract}
Introduction: Combined liver and kidney transplantation (CLK) has been shown to be a good alternative for patients with concomitant diagnosis of chronic kidney disease (CKD) and end-stage liver disease. Some studies have also shown immunological benefits from combined transplantation with decreased rates of kidney graft rejection.

Objective: The objective of this study was to describe the indications and clinical outcomes of CLK transplant recipients in a highly complex hospital.

Materials and Methods: CLK transplant patients were selected from the institutional transplant registry (Trenal) from 2000 to 2011. Demographic and clinical characteristics were described and survival of patients and grafts were estimated with the Kaplan-Meier method.

Results: Over a period of 11 years, 16 CLK transplants were performed. This was $1.51 \%$ of kidney transplants and $3.48 \%$ of liver transplants done in the institution during this period. Most recipients were male (10/16). The median age was 56.5 years. The median MELD was 17 (IQR: 12.5 to 20.5 , range: 8 to 24$)$. The most frequent diagnosis was liver cirrhosis due to NASH (4/16). All patients had been diagnosed with CKD: four cases were secondary to diabetes mellitus. The most common indications for transplants were difficult to manage ascites, recurrent encephalopathy and malnutrition. The average liver cold ischemia time was 7.3 hours, and the average kidney cold ischemia time 9.6 hours. The five-year liver graft survival rate was $87.5 \%$, and the five-year kidney graft survival rate was $67.3 \%$. Four patients died: two as the result of sepsis and two as the result of malignancy.

Conclusion: CLK transplantation results at the Fundación Valle del Lili have been satisfactory and comparable to those reported by other transplant centers.

\section{Keywords}

Combined liver-kidney transplant, survival, graft rejection.
\end{abstract}

proteger el injerto renal, y de disminuir la tasa de rechazo agudo del injerto renal $(4,5)$. Además, el trasplante CLK contribuye a mejorar la calidad de vida, ya que permite que un paciente con enfermedad renal crónica (ERC) no dependa de la diálisis, con el beneficio adicional en términos de costos que esto conlleva (6). La Fundación Valle del Lili es una clínica que cuenta con una amplia experiencia en trasplante de órganos sólidos desde 1995. En el año 2000 se hizo el primer trasplante CLK y desde entonces se han intervenido 16 pacientes; El propósito de este estudio fue describir nuestra experiencia en el manejo de pacientes con trasplante combinado de hígado y riñón.

\section{PACIENTES Y MÉTODOS}

Los datos del registro de trasplante renal y hepático se obtuvieron de la institución (TRENAL). Este es un registro que se lleva desde 2010 e incluye la información de los trasplantes renales hechos desde 1995 en la Fundación Valle del Lili. Se incluyeron los pacientes sometidos a trasplante CKL entre el año 2000 y el 2011; de forma sistemática se recolectó la información demográfica y clínica relacionada con la indicación del trasplante, las complicaciones quirúrgicas y médicas tempranas (en los primeros tres meses postrasplante) y tardías (después de tres meses postrasplante). El ingreso a la lista de espera estuvo determinado por la evaluación clínica pretrasplante y sometido a discusión en la junta de trasplante de la institución. En el momento del trasplante la selección del receptor se hizo a partir de la compatibilidad del grupo sanguíneo. No se hicieron pruebas de citotoxicidad con el donante previo al trasplante.

La terapia de inmunosupresión se estableció según las condiciones inmunológicas y clínicas de cada paciente. Los pacientes recibieron un inhibidor de calcineurina $(\mathrm{n}=15)$, micofenolato $(\mathrm{n}=13)$, azatioprina $(\mathrm{n}=2)$ y esteroides $(\mathrm{n}=16)$. En tres casos se utilizó inducción con anticuerpos monoclonales. Los pacientes asistieron regularmente a control mensual como parte del programa de seguimiento postrasplante. Se describieron las características demográficas y clínicas. Se usaron medianas y rango intercuartil (RIC) o promedio \pm la desviación estándar (DE) para las variables cuantitativas y proporciones para las variables cualitativas. La sobrevida del paciente y de los injertos renal y hepático se calculó con el método de Kaplan Meyer, a través del software STATA 12.

\section{RESULTADOS}

En la institución entre 2000 y 2011 se hicieron 1059 trasplantes de riñón y 459 trasplantes de hígado. En este mismo período se hicieron 16 trasplantes CLK, un 1,51\% de todos los trasplantes de riñón y $3,48 \%$ de todos los trasplantes de hígado. Diez pacientes fueron hombres y 6 mujeres. La mediana de la edad al momento del trasplante fue de 56,5 
años (RIC: 50,7-64,5; rango 3-69), incluidos dos pacientes pediátricos de 3 y 7 años. Las etiologías de la enfermedad hepática y renal se describen en la Tabla 1 . A un paciente se le hizo biopsia hepática pretrasplante, que mostró cirrosis biliar primaria. A tres pacientes se les hizo biopsia renal pretrasplante: dos mostraron glomerulonefritis proliferativa y la tercera, riñón terminal.

Dentro de los antecedentes patológicos, $6 / 16$ pacientes presentaron diabetes mellitus tipo $2,4 / 16$ tuvieron dislipidemia, $3 / 16$ hipertensión arterial y $3 / 16$ historia de malignidad. La mediana del MELD (Model for end-stage Liver Disease) fue de 17 (RIC: 12,5-20,5, rango 8-240029. En uno de los dos pacientes pediátricos, el cálculo del PELD (Pediatric End-Stage Liver Disease) fue 1, con diagnóstico de fibrosis hepática congénita. En el otro paciente no se realizó esta escala ya que el diagnóstico que indicó el trasplante fue hiperoxaluria primaria. El promedio de tiempo de isquemia en frío del hígado fue de 7,3 horas con una DE $\pm 2,3$ (rango 4-12) y el del riñón de 9,6 horas con una DE \pm 2,2 (rango 6-12). El tiempo promedio en lista de espera fue de 157 días, con una $\mathrm{DE} \pm 112$ (rango 1-422). Hubo 2/16 pacientes trasplantados en urgencia cero. La mediana del tiempo de seguimiento postrasplante fue 35 meses (RIC: 18-48, rango 0,07-156). Tres pacientes recibieron inducción con anticuerpos monoclonales, uno de ellos recibió daclizumab y los otros dos basiliximab.

La Tabla 2 muestra las complicaciones quirúrgicas y médicas. En 11 pacientes (68\%) se documentó al menos una complicación, entre las que se incluyen: complicaciones biliares tempranas ( 3 pacientes), complicaciones biliares tardías (5 pacientes), sangrado ( 2 pacientes) e infección por citomegalovirus ( 1 paciente). Tres pacientes fueron diagnosticados con rechazo agudo leve del injerto hepático; dos de estos se presentaron entre los 3 a 9 meses postrasplante, y uno a los 10 años postrasplante. Este diagnóstico se hizo por signos clínicos asociados con alteración del perfil hepático, confirmándose con biopsia en dos pacientes y en uno por la respuesta al tratamiento empírico. Un paciente de 7 años de edad fue diagnosticado con rechazo agudo del injerto renal por biopsia, a los 3 meses postrasplante. Todos los eventos de rechazo agudo se trataron con esteroides y en todos los casos respondieron al tratamiento. La sobrevida, tanto del paciente como de los injertos hepático y renal, fue de $87,5 \%$ al año y $67,3 \%$ a los 5 años (Figura 1). Dos pacientes murieron por sepsis, uno de ellos en el postoperatorio inmediato y el otro al tercer mes postrasplante. Dos pacientes murieron por cáncer después del primer año postrasplante. De estos, uno fue un linfoma de células B y el otro, recurrencia de hepatocarcinoma. De los 12 pacientes que continúan con los injertos funcionantes, ninguno ha requerido retrasplante.

\section{DISCUSIÓN}

El programa de trasplante combinado de hígado y riñón de la Fundación Valle del Lili presenta una incidencia de

Tabla 1. Características de los receptores de trasplante CLK en la Fundación Valle del Lili, Cali, Colombia

\begin{tabular}{cclll}
\hline Paciente & Edad (años) & Sexo & \multicolumn{1}{c}{ Nefropatía } & \multicolumn{1}{c}{ Hepatopatía } \\
\hline 1 & 65 & M & Glomerulonefritis & Cirrosis HCV + Hepatocarcinoma \\
2 & 68 & M & Nefropatía DM & Cirrosis por NASH \\
3 & 35 & M & Nefropatía lgA & Cirrosis por NASH + Hemocromatosis \\
4 & 55 & M & Desconocida & Hepatocarcinoma + Cirrosis criptogénica \\
5 & 50 & M & Desconocida & Cirrosis por alcohol \\
6 & 69 & F & Nefropatía DM & Cirrosis HCV \\
7 & 60 & M & Desconocida & Cirrosis autoinmune \\
8 & 66 & F & Desconocida & Cirrosis criptogénica \\
9 & 54 & F & Nefropatía DM & Cirrosis por NASH \\
10 & 57 & F & Nefropatía DM & Cirrosis HCV \\
11 & 3 & F & Riñones poliquísticos & Fibrosis hepática congénita \\
12 & 58 & M & Nefrotoxicidad por medicamentos & Cirrosis por NASH \\
13 & 7 & M & Hiperoxaluria primaria & Hiperoxaluria primaria \\
14 & 52 & M & Desconocida & Cirrosis biliar primaria \\
15 & 55 & M & Glomerulonefritis & Cirrosis por alcohol \\
16 & 63 & F & Desconocida & Colangitis crónica \\
\hline
\end{tabular}


rechazo agudo del injerto renal baja, con complicaciones similares a las reportadas por otros grupos de trasplante (véase Tabla 2) $(2,7,8)$. En la literatura no se han descrito mayores complicaciones asociadas con el trasplante combinado de hígado riñón frente al de hígado aislado (9-13). En nuestra población de estudio, tanto la sobrevida del paciente como la sobrevida del injerto hepático y renal a 5 años fueron del $67,3 \%$. Estos datos son similares a los reportados en otras series, incluyendo el registro de la UNOS $(7,11,14-17)$, donde la sobrevida fue de $58 \%$. La sobrevida del injerto hepático y renal censurada por muerte a 5 años fue del $100 \%$. De los 16 trasplantes combinados de hígado y riñón de nuestra institución, solo un paciente pediátrico presentó rechazo agudo del injerto renal $(6,7 \%)$ al tercer mes postrasplante. Dos pacientes $(12,5 \%)$ presentaron rechazo agudo leve del injerto hepático en el primer año. De acuerdo con nuestras propias estadísticas, en los mismos períodos de tiempo postrasplante (datos no publicados); estas incidencias de rechazo agudo son menores que las del grupo de receptores de solo trasplante renal (20\%) o hepático (21\%).

Tabla 2. Complicaciones quirúrgicas y médicas postrasplante tempranas y tardías en receptores de trasplante CLK en la Fundación Valle del Lili, Cali, Colombia

\begin{tabular}{ll}
\hline \multicolumn{1}{c}{ Complicaciones postrasplante } & n \\
\hline Quirúrgicas - Tempranas & 2 \\
Fístula biliar & 2 \\
Sangrado & 1 \\
Trombosis de la arteria hepática & 1 \\
Obstrucción ureteral & 1 \\
Isquemia con necrosis de colon izquierdo & 1 \\
Nefrotoxicidad a la ciclosporina & \\
Quirúrgicas - Tardías & 5 \\
Estenosis de la vía biliar & 1 \\
Eventración & \\
Médicas - Tardías & 3 \\
DM novo & 3 \\
HTA postrasplante & 1 \\
Nefrotoxicidad a la ciclosporina & 1 \\
Recurrencia de hepatocarcinoma & 1 \\
Malignidad (linfoma de células B) & 1 \\
Trombosis venosa profunda/TEP & 1 \\
Enfermedad hepática venoclusiva & 1 \\
Infección por citomegalovirus & \\
\hline
\end{tabular}

Creput y colaboradores describieron que la incidencia de rechazo agudo, tanto para el injerto renal como el injerto hepático, en pacientes con trasplante simultáneo de hígado y riñón, fue menor que en pacientes con trasplante renal o hepático solo (17). Así mismo, Fong y colaboradores reportaron que al comparar la incidencia de rechazo agudo del injerto renal en pacientes con trasplante CLK frente a trasplante renal solo, dicha incidencia fue menor en los pacientes con trasplante CLK (14). Al revisar otros reportes relacionados encontramos que la incidencia de rechazo agudo del injerto renal al año en pacientes que reciben trasplante renal postrasplante hepático está alrededor del 18\%, mientras que en los pacientes con trasplante combinado hígado y riñón fue $11,8 \%$ (2). Esto puede explicarse por las publicaciones que describen el injerto hepático con un rol protector, no solo en la fase inicial postrasplante, sino también en el mantenimiento a largo plazo de la función del injerto renal, mediante la reducción de los niveles de reactividad antidonante específico (3). Esto, como posible resultado de la mediación de activación de la enzima indolamina 2-3 dioxigenasa (IDO) (18).

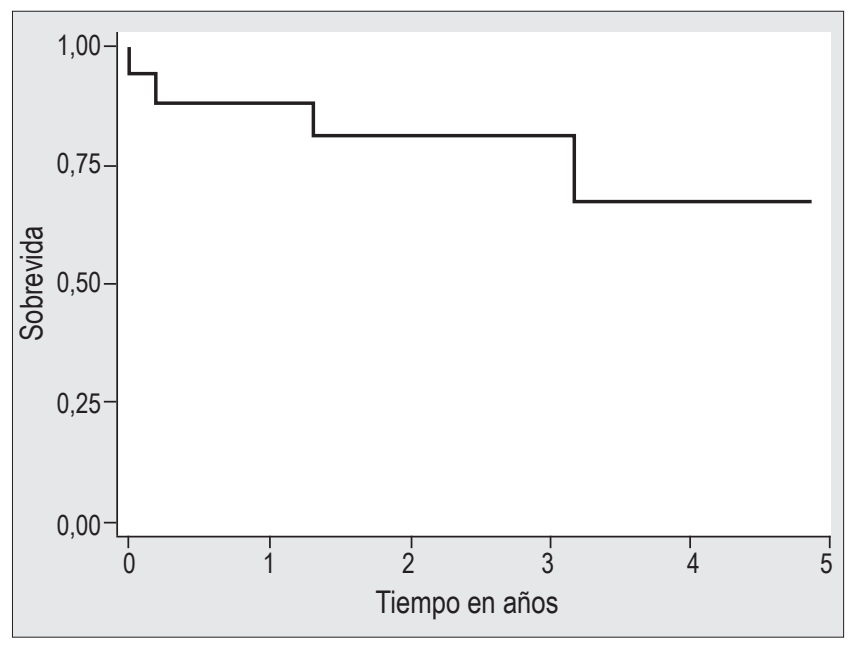

Figura 1. Sobrevida del paciente y de los injertos hepático y renal en receptores de trasplante CLK en la Fundación Valle del Lili, Cali, Colombia.

\section{CONCLUSIONES}

Para mejorar la eficiencia, disminuir la mortalidad de los pacientes en lista de espera y hacer más objetivo el ingreso a la lista para trasplante se creó el puntaje MELD, el cual incorpora la función renal para su cálculo. Su uso ha aumentado el número de trasplantes combinados de hígado y riñón. Después de esta medida se ha encontrado una disminución de ERC en receptores de trasplante hepático, menor tiempo de estancia en UCI y menos costos en salud, además de un efecto de protección inmunológica del hígado sobre el injerto renal en los pacientes llevados a trasplante combinado (2). Lo que está claro es que el uso de inhibidores de calcineurina, las interacciones farmacológicas y el riesgo de infecciones después del trasplante hepá- 
tico hacen que la enfermedad renal avance, y los resultados del trasplante combinado de hígado y riñón aparentemente son mejores que los resultados de un trasplante secuencial primero de hígado y después de riñón $(2,9)$.

La indicación del trasplante simultáneo de hígado-riñón es clara cuando el paciente se encuentra en diálisis, con enfermedad renal no reversible; en pacientes con tasa de filtración glomerular (TFG) menor a $40 \mathrm{~mL} / \mathrm{min}$, aunque otros autores lo han definido con TFG menor a $30 \mathrm{~mL} /$ min $(16,19)$. Independiente de la TFG establecida, la falla renal crónica no reversible es una indicación para trasplante combinado de hígado riñón dadas las consecuencias nefrotóxicas del uso de inhibidores de calcineurina postrasplante de hígado sobre la función de los riñones nativos $(9,12,15)$. Ha sido de especial interés definir la posibilidad de recuperación de la función de los riñones nativos posterior a la realización de trasplante hepático aislado o CLK, para lo cual se han llevado a cabo estudios que incluyen la realización de gammagrafía renal con MAG3 o DTPA pre y postrasplante, así como la utilización de biomarcadores diferentes a la creatinina sérica, que permitan decidir mejor la asignación de los órganos disponibles para trasplante $(20,21)$. En esta serie no se documentaron posibles cambios en la función de los riñones nativos postrasplante. Este es un tema ideal para tener en cuenta en estudios posteriores. El trasplante combinado de hígado y riñón en este centro tiene resultados clínicos comparables a los reportados en centros de otros países con más experiencia y ha mostrado ser una buena opción terapéutica para pacientes que cursan simultáneamente con enfermedad hepática terminal y enfermedad renal crónica.

\section{REFERENCIAS}

1. Margreiter R, Kramar R, Huber C, Steiner E, Niederwieser $\mathrm{D}$, Judmaier $\mathrm{G}$, et al. Combined liver and kidney transplantation. Lancet. 1984;1(8385):1077-8.

2. Simpson N, Cho YW, Cicciarelli JC, Selby RR, Fong T-L. Comparison of renal allograft outcomes in combined liverkidney transplantation versus subsequent kidney transplantation in liver transplant recipients: Analysis of UNOS Database. Transplantation. 2006;82(10):1298-303.

3. Eason JD, Gonwa TA, Davis CL, Sung RS, Gerber D, Bloom RD. Proceedings of Consensus Conference on Simultaneous Liver Kidney Transplantation (SLK). Am J Transplant. 2008;8(11):2243-51.

4. Olausson M, Mjörnstedt L, Nordén G, Rydberg L, Mölne J, Bäckman L, et al. Successful combined partial auxiliary liver and kidney transplantation in highly sensitized cross-match positive recipients. Am J Transplant. 2007;7(1):130-6.

5. Alqurashi S, Alsayyari AA, Abdullah K, Alwan A, Hajeer AH. Combined liver and kidney transplantation in a highly sensitized and positively cross-matched patient. Saudi J Kidney Dis Transpl. 2011;22(4):757-60.
6. Rosselli D. CS3 Un modelo de costo-utilidad del trasplante renal en Colombia. Value in Health. 2009;12(7):A484.

7. JL GB. Evolución del injerto renal en los pacientes con trasplante hepático asociado. En: Portillo Martín JA BDR, Zubillaga Guerrero S, Ramos Barselo E, Campos Sañudo JA, ed., Actas Urol Esp. 2008:220-4.

8. Fung J, Makowka L, Tzakis A, Klintmalm G, Duquesnoy R, Gordon $\mathrm{R}$, et al. Combined liver-kidney transplantation: analysis of patients with preformed lymphocytotoxic antibodies. Transplant Proc. 1988;20(1 Suppl 1):88-91.

9. Martin EF, Huang J, Xiang Q, Klein JP, Bajaj J, Saeian K. Recipient survival and graft survival are not diminished by simultaneous liver-kidney transplantation: an analysis of the united network for organ sharing database. Liver Transpl. 2012;18(8):914-29.

10. Ma Y, Wang G, He X, Li Q, Li J, Zhu X, et al. Simultaneous liver and kidney transplantation: analysis of a single-center experience. Chin Med J. 2010;123(10):1259-63.

11. Ruiz R, Kunitake H, Wilkinson AH, Danovitch GM, Farmer DG, Ghobrial RM, et al. Long-term analysis of combined liver and kidney transplantation at a single center. Arch Surg. agosto de 2006;141(8):735-741-742.

12. Veras FJ de O, Coelho GR, Feitosa-Neto BA, Cerqueira JBG, Garcia RCFG, Garcia JHP. Combined liver-kidney transplantation: experience at a Brazilian university hospital. Arq Bras Cir Dig. 2014;27(1):53-5.

13. González MR, Ramírez P, Cascales P, Domingo J, López $\mathrm{MD}$, Rios A, et al. Thirteen cases of liver-kidney transplantation. Transplant Proc. 2010;42(8):3162-3.

14. Fong T-L, Bunnapradist S, Jordan SC, Selby RR, Cho YW. Analysis of the United Network for Organ Sharing database comparing renal allografts and patient survival in combined liver-kidney transplantation with the contralateral allografts in kidney alone or kidney-pancreas transplantation. Transplantation. 2003;76(2):348-53.

15. Xing T, Zhong L, Chen D, Peng Z. Experience of combined liver-kidney transplantation for acute-on-chronic liver failure patients with renal dysfunction. Transplant Proc. 2013;45(6):2307-13.

16. Haad CR, Rodriguez-BenotA, Martinez-Vaquera S, NavarroCabello MD, Aguera-Morales ML, Ruiz de Mier MVP, et al. Combined liver-kidney transplantation: survey of a single center in Spain. Transplant Proc. 2013;45(10):3640-3.

17. Creput C, Durrbach A, Samuel D, Eschwege P, Amor M, Kriaa $F$, et al. Incidence of renal and liver rejection and patient survival rate following combined liver and kidney transplantation. Am J Transplant. 2003;3(3):348-56.

18. Ingelsten $M$, Gustafsson K, Oltean M, Karlsson-Parra A, Olausson M, Haraldsson B, et al. Is indoleamine 2,3-dioxygenase important for graft acceptance in highly sensitized patients after combined auxiliary liver-kidney transplantation? Transplantation. 2009;88(7):911-9.

19. Hanish SI, Samaniego M, Mezrich JD, Foley DP, Leverson GE, Lorentzen DF, et al. Outcomes of simultaneous liver/ kidney transplants are equivalent to kidney transplant alone: a preliminary report. Transplantation. 2010;90(1):52-60. 
20. Francis JM, Palmer MR, Donohoe K, Curry M, Johnson SR, Karp SJ, et al. Evaluation of native kidney recovery after simultaneous liver-kidney transplantation. Transplantation. 2012;93(5):530-5.
21. Levitsky J, Baker T, Ahya SN, Levin ML, Friedewald J, Gallon $\mathrm{L}$, et al. Outcomes and native renal recovery following simultaneous liver-kidney transplantation. Am J Transplant. 2012;12(11):2949-57. 